\title{
Gamificación personalizada para fortalecer aprendizajes significativos de la asignatura matemática
}

\author{
Carlos Luis Sánchez-Pacheco a
}

Resumen - El objetivo de la presente investigación es demostrar que la aplicación de gamificación fortalece el aprendizaje significativo de la asignatura Matemática. La investigación mixta cuya población fueron los estudiantes de bachillerato de la ciudad de Guayaquil. Los datos se recolectaron mediante un cuestionario de Google Forms. Sobre el conocimiento de Gamificación, se encontró que hay estudiantes que manejan la estrategia sin conocer sus fundamentos teóricos, hay estudiantes que tienen poco conocimiento y hay quienes solo se enteraron de la técnica.

Palabras clave - Aprendizaje significativo, Gamificación, Matemática, Innovación, TIC.

\begin{abstract}
The objective of this research is to demonstrate that the application of gamification strengthens the meaningful learning of the mathematics subject. The mixed research whose population was high school students from the city of Guayaquil. The data was collected through a Google Forms questionnaire. Regarding the knowledge of Gamification, it was found that there are students who handle the strategy without knowing its theoretical foundations, there are students who have little knowledge and there are those who only found out about the technique.
\end{abstract}

Keywords - Meaningful learning, Gamification, Math, Innovation, ICT.

\section{CÓMO CITAR \\ HOW TO CITE:}

Sánchez-Pacheco, C. L. (202I).

Gamificación personalizada para

fortalecer aprendizajes

significativos de la asignatura matemática. Interconectando

Saberes, (I2), 29-37.

https://doi.org// 0.25009/is.v0il2 .2680

Recibido: 25 de agosto de 2020 Aceptado: 5 de mayo de 2021 Publicado: 20 de julio de 2021

a Sociedad de Investigación Pedagógica Innovar, Colombia. E-mail: arlossanchez21@hotmail.com 


\section{INTRODUCCIÓN}

La gamificación es una técnica persuasiva que busca aprovechar el interés de los usuarios en los juegos para aumentar su participación y aceptación de tareas o entornos que inicialmente podrían no atraerlos o incluso desagradarlos (Borges, y otros, 2017). Para esto, se utilizan pequeños elementos de los juegos junto con el proceso de realización de la tarea, proporcionando al usuario la sensación de que al cumplirlo está participando en un juego (Andrade, Mizoguchi, \& Isotani, 2016; Deterding, 20I2; Kapp, 20I2).

Por lo tanto, la Gamificación propone no crear un juego completo en el que la actividad sea una de sus etapas, sino utilizar e incorporar parte del elemento que hace divertidos los juegos como la competencia, la cooperación y las recompensas en el juego. Tarea propia que el usuario debe realizar para hacerlo más interesante (Deterding, 2012; Kapp, 2012; Zichermann \& Cunningham, 20II).

La gamificación en la educación se ha utilizado tanto dentro como fuera del aula, haciendo hincapié en los esfuerzos para aumentar la participación de los usuarios en los Entornos Virtuales de Aprendizaje (EVA). Por lo tanto, plataformas educativas como Khan Academy, My Tutor y Duolingo han insertado varios elementos del juego, como tablas de clasificación, puntos y misiones para alentar a los estudiantes a mantenerse involucrados por más tiempo.

De acuerdo con Pedro, Lopes, Prates, Vassileva, y Isotani (20I5), el diseño de gamificación puede afectar a los usuarios de diferentes maneras, dependiendo de sus características, como su género, dominio de la actividad y atributos psicológicos, sugiriendo que los estudios de la gamificación en el contexto del aprendizaje deben considerar la diferencia de género para sacar mejores conclusiones sobre su impacto en motivación y rendimiento de aprendizaje

Aunque pudimos identificar a partir de la evidencia de los estudios anteriores que sugiere la importancia de considerar las características del usuario al diseñar entornos y actividades, la implementación de la gamificación actualmente se centra principalmente en enfoques únicos para todos, es decir no considera las diferencias entre los usuarios al proponer elementos 0 estrategias de juego para la gamificación de entornos, ni se adaptan a los usuarios en tiempo de ejecución (Borges, y otros, 2017; Andrade, Mizoguchi, \& Isotani, 2016).

Aunque pudimos identificar a partir de la evidencia de los estudios anteriores que sugiere la importancia de considerar las características del usuario al diseñar entornos y actividades, la implementación de la gamificación actualmente se centra principalmente en enfoques únicos para todos, es decir no considera las diferencias entre los usuarios al proponer elementos o estrategias de juego para la gamificación de entornos, ni se adaptan a los usuarios en tiempo de ejecución (Borges, y otros, 2017; Andrade, Mizoguchi, \& Isotani, 2016).

Por lo tanto, en esta investigación, consideramos que la comprensión de la personalización y los enfoques centrados en el usuario en el dominio de la gamificación, son críticos para lograr resultados consistentes cuando se usa como estrategia motivacional. Cuyo objetivo es comprobar que la aplicación de gamificación personalizada fortalecerá el aprendizaje significativo de las matemáticas en los estudiantes de bachillerato, es decir, entornos cuyas opciones de acceso dependen totalmente del estudiante. Creemos en esta investigación que la personalización de 
la gamificación puede afectar positivamente la participación espontánea de los estudiantes en plataformas abiertas, aumentando sus interacciones en el sistema, su tiempo de permanencia y la tasa de retorno del usuario.

Por todas estas consideraciones, en este trabajo, formulamos el siguiente problema: ¿En qué medida la gamificación personalizada fortalecerá los aprendizajes significativos de la asignatura Matemática en los estudiantes de bachillerato de la ciudad de Guayaquil?

El objetivo general, es examinar la evidencia existente sobre la incidencia del uso de softwares gamificados en el mejoramiento del rendimiento de los estudiantes en el aprendizaje de las matemáticas.

Específicamente se propone identificar el nivel de participación del usuario cuando se usa el modelado de gamificación personalizado sobre el modelado no personalizado; aplicar test que facilite la identificación de los elementos de los juegos dados: tipos de personalidades y jugadores de los colaboradores $y$ las adversidades reportadas en el entorno colaborativo; comprobar el cambio generado en el aprendizaje significativo mediante la utilización de gamificación personalizada.

\section{MATERIAL Y MÉTODOS}

En cuanto al término gamificación, proviene del inglés Gamification, es un concepto relativamente nuevo, que es el uso de elementos de diseño de juegos en entornos ajenos al juego, como las redes sociales, las aplicaciones de atención médica, los sistemas educativos y las empresas, para motivar a las personas y cambiar comportamientos (Deterding, 2012; Hamari, Koivisto, \& Sarsa, 20I4; Kapp, 2012)
Sin embargo, este concepto no debe confundirse con juegos educativos o juegos serios (Deterding, 2012), ya que la Gamificación propone el uso de parte de los conceptos aplicados durante el desarrollo de juegos en actividades ajenas al juego, tratando de incorporar algunos de los elementos que las personas encuentran interesantes durante su entretenimiento en las actividades cotidianas para hacerlos más atractivos (Deterding, 20I2; Kapp, 20I2). A su vez, "Juegos serios" o "Juegos de propósito" son juegos completos que tienen como objetivo ofrecer contenido específico o capacitar a los usuarios para una actividad en particular (Deterding, 20I2).

Para la educación, mantener a los estudiantes motivados mientras usan software educativo y entornos virtuales de enseñanza y aprendizaje ha sido uno de los principales desafíos de la comunidad de Computación Aplicada a la Educación (Lozada-Ávila \& BetancurGómez, 2017).

Por lo tanto, la propuesta de aumentar la motivación y el compromiso de los estudiantes a través de la Gamificación ha despertado un gran interés en los investigadores de la comunidad $y$, aunque las prácticas de implementación aún están mal establecidas, el número de encuestas ha aumentado considerablemente en los últimos años (Nah, Zeng, Telaprolu, Ayyappa, \& Eschenbrenner, 20I4; Hamari, Koivisto, \& Sarsa, 20I4). Se puede observar esto a través de la cantidad de artículos analizados en las diversas revisiones de literatura que se han publicado buscando este fenómeno creciente.

El aprendizaje significativo es el concepto central de la teoría de Ausubel (1976). El aprendizaje significativo es una teoría cognitiva desarrollada por el psicólogo estadounidense David Paul Ausubel, que era 
profesor emérito en la Universidad de Columbia en Nueva York, y luego fue refinado junto con Novak y Hanesian. En este enfoque, el libro de Ausubel, adquisición y retención del conocimiento: una perspectiva cognitiva, y algunas producciones de otros autores que resaltan los conceptos de esta teoría se utilizaron como referencia.

En la propuesta de Ausubel (1976), el aprendizaje significativo se conceptualiza como "el mecanismo humano, por excelencia, para adquirir y almacenar la gran cantidad de ideas e información representadas en cualquier campo del conocimiento". Moreira (1997) especifica cómo "el proceso por el cual la nueva información (nuevo conocimiento) se relaciona de manera no arbitraria y sustantiva (no literal) a la estructura cognitiva del alumno", destacando así los dos puntos clave de la teoría: la no arbitrariedad y la sustantividad.

\section{TIPO DE ESTUDIO}

El presente estudio tiene un diseño cuasi experimental, como lo indica Ñaupas, Mejía, Novoa, y Villagómez (2014) este diseño trabaja con grupos no aleatorizados, su validez es pequeña porque no hay control sobre las variables extrañas (p338). Consistió en la aplicación de un pretest a los grupos (experimental y control), para tener el referente sobre el aprendizaje significativo; para luego aplicar la intervención, en el caso de gamificación personalizada al grupo experimental para después aplicar el post test a los dos grupos, el grupo control sirve para analizar si la personalización de la gamificación tiene un efecto sobre el aprendizaje significativo.

\section{POBLACIÓN Y MUESTRA}

La población está determinada por los estudiantes del nivel de Bachillerato de la ciudad de Guayaquil, tal como se indica en el título de la investigación. A decir de Carrasco (20I5), la población se trata del "conjunto de todos los elementos que pertenecen al ámbito espacial donde se desarrolla el trabajo de investigación" (p237); en la presente investigación se trabajó como muestra a los estudiantes del Bachillerato Técnico en Informática del Distrito Ximena 2, los cuales son asignados como el grupo experimental (GE); y el segundo grupo será asignado como el grupo control (GC) (ver Tabla I).

\section{Tabla I}

Muestra de estudiantes del Bachillerato Técnico en Informática

\begin{tabular}{lc}
\hline \multicolumn{1}{c}{$\begin{array}{c}\text { Estudiantes del Bachillerato } \\
\text { Técnico en Informática }\end{array}$} & Muestra \\
\hline Grupo de Control & 34 \\
Grupo experimental & 32 \\
\hline Total & 66
\end{tabular}

\section{INSTRUMENTO}

El instrumento de investigación que se utilizó en el proceso de acopio de datos, en el presente estudio se realizó la aplicación de un formulario web para validar la asignación de elementos del juego mediante tipo de personalidad y tipo de jugador.

Con el objetivo de realizar pruebas estadísticas para validar el marco propuesto, se utilizará el modelo de Likert para la evaluación de las 4 dimensiones de la gamificación en la educación. El modelo de ítem Likert se utiliza para medir aspectos del comportamiento social, como los rasgos de personalidad y carácter, desde un punto de vista matemático, por ejemplo, estadísticas descriptivas, pruebas estadísticas 
paramétricas y no paramétricas (Likert, 1932; Clason \& Dormody, 1994). Este modelo se utilizó para medir la cantidad de elementos del juego que estimulan a los participantes en una escala de 4 niveles, donde los elementos uno (I) y cuatro (4) representan, respectivamente, el desacuerdo total y el acuerdo total.

\section{RESULTADOS}

El estudio se realizó con la participación de 66 estudiantes del Bachillerato Técnico en Informática del Distrito Ximena 2. El 46,84 \% de la muestra estuvo conformada por mujeres y el 53,16\% por hombres. Las edades de los participantes oscilan entre los 16 y 18 años. De acuerdo con el nivel en el que estudian los participantes son del nivel de bachillerato.

Todos contaron con acceso a Internet desde sus casas con situaciones homogéneas en cuanto al acceso a internet, hecho fundamental para poder desarrollar a cabo la experiencia.

En el pretest: Observamos que los resultados iniciales de los saberes previos de los estudiantes del bachillerato, tanto en el grupo control y experimental presentan condiciones iniciales semejantes, es decir no existen diferencias significativas (ver Tabla 2).

En el post test: Después de la aplicación del aula virtual gamificada existe una varianza en el resultado de Saberes previos. En el grupo control el $76.47 \%$ permanece en el nivel deficiente. En contraste, en el grupo experimental el $40.62 \%$ de estudiantes logra el nivel medio; asimismo el $25 \%$ alcanza el nivel alto. A esto se agrega que solo el $34.38 \%$ muestra las dificultades iniciales (ver Tabla 2).

En el pretest: En esta dimensión observamos que el grupo control y experimental presentan deficiencia Construcción de los nuevos conocimientos. Ambos grupos requieren el uso estrategias para la construcción de los nuevos conocimientos (ver Tabla 3).

En el post test: Los dos grupos presentan significativas diferencias. El grupo control se mantiene en los porcentajes. El grupo experimental ha logrado mejoría en la construcción de nuevos conocimientos porque presenta el $37.5 \%$ de deficiencia, mientras que el $31.25 \%$ presenta nivel medio y alto (ver Tabla 3 ).

\section{Tabla 2}

Nivel de Saberes previos

\begin{tabular}{lccccccccc}
\hline & & \multicolumn{4}{c}{ Grupo Control } & \multicolumn{3}{c}{ Grupo Experimental } \\
& Rango & \multicolumn{2}{c}{ Pretest } & \multicolumn{2}{c}{ Post test } & \multicolumn{2}{c}{ Pretest } & \multicolumn{2}{c}{ Post test } \\
& & $\mathbf{n}$ & $\%$ & $\mathbf{n}$ & $\%$ & $\mathbf{n}$ & $\%$ & $\mathbf{n}$ & $\%$ \\
\hline Deficiente & $01-07$ & 27 & 79.41 & 26 & 76.47 & 24 & 75.00 & 11 & 34.38 \\
Medio & $08-14$ & 5 & 14.71 & 5 & 14.71 & 6 & 18.75 & 13 & 40.62 \\
Alto & $15-20$ & 2 & 5.88 & 3 & 8.82 & 2 & 6.25 & 8 & 25.00 \\
\hline Total & & 34 & 100.00 & 34 & 100.00 & 32 & 100.00 & 32 & 100.00 \\
\hline
\end{tabular}


Tabla 3

Nivel de construcción de los nuevos conocimientos

\begin{tabular}{lccccccccc} 
& & \multicolumn{3}{c}{ Grupo Control } & \multicolumn{3}{c}{ Grupo Experimental } \\
& Nivel & Rango & \multicolumn{2}{c}{ Pretest } & \multicolumn{2}{c}{ Post test } & \multicolumn{2}{c}{ Pretest } & \multicolumn{2}{c}{ Post test } \\
& & $\mathbf{n}$ & $\%$ & $\mathbf{n}$ & $\%$ & $\mathbf{n}$ & $\%$ & $\mathbf{n}$ & $\%$ \\
\hline Deficiente & $01-07$ & 28 & 82.35 & 27 & 79.40 & 29 & 90.63 & 12 & 37.50 \\
Medio & $08-14$ & 2 & 5.88 & 3 & 8.82 & 3 & 9.37 & 10 & 31.25 \\
Alto & $15-20$ & 2 & 5.88 & 2 & 5.88 & 0 & 0.00 & 10 & 31.25 \\
\hline Total & & 34 & 100.00 & 34 & 100.00 & 32 & 100.00 & 32 & 100.00 \\
\hline
\end{tabular}

\section{Tabla 4}

Nivel de transferencia de aprendizaje

\begin{tabular}{lccccccccc}
\hline & & \multicolumn{4}{c}{ Grupo Control } & \multicolumn{3}{c}{ Grupo Experimental } \\
& Rango & \multicolumn{2}{c}{ Pretest } & \multicolumn{2}{c}{ Post test } & \multicolumn{2}{c}{ Pretest } & \multicolumn{2}{c}{ Post test } \\
& & $\mathbf{n}$ & $\%$ & $\mathbf{n}$ & $\%$ & $\mathbf{n}$ & $\%$ & $\mathbf{n}$ & $\%$ \\
\hline Deficiente & $01-07$ & 30 & 88.24 & 30 & 88.24 & 29 & 90.63 & 14 & 43.75 \\
Medio & $08-14$ & 4 & 11.76 & 4 & 11.76 & 3 & 9.37 & 12 & 37.50 \\
Alto & $15-20$ & 0 & 0.00 & 0 & 0.00 & 0 & 0.00 & 6 & 18.75 \\
\hline Total & & 34 & 100.00 & 34 & 100.00 & 32 & 100.00 & 32 & 100.00 \\
\hline
\end{tabular}

\section{Tabla 5}

Nivel de gamificación en el aula

\begin{tabular}{lccccccccc}
\hline & & \multicolumn{3}{c}{ Grupo Control } & \multicolumn{3}{c}{ Grupo Experimental } \\
& Rivel & & \multicolumn{2}{c}{ Pretest } & \multicolumn{2}{c}{ Post test } & \multicolumn{2}{c}{ Pretest } & \multicolumn{2}{c}{ Post test } \\
& & $\mathbf{n}$ & $\%$ & $\mathbf{n}$ & $\%$ & $\mathbf{N}$ & $\%$ & $\mathbf{n}$ & $\%$ \\
\hline Deficiente & $01-07$ & 16 & 47.06 & 15 & 44.12 & 17 & 53.13 & 5 & 15.63 \\
Medio & $08-14$ & 13 & 38.24 & 14 & 41.18 & 12 & 37.50 & 18 & 56.25 \\
Alto & $15-20$ & 5 & 14.70 & 5 & 14.70 & 3 & 9.37 & 9 & 28.12 \\
\hline Total & & 34 & 100.00 & 34 & 100.00 & 32 & 100.00 & 32 & 100.00 \\
\hline
\end{tabular}

En el pretest: Los grupos tienen características deficientes para la transferencia de aprendizaje. Ambos grupos, requieren de intervención (ver Tabla 4).

En el post test: En el grupo experimental se observa que la transferencia de aprendizaje después de la intervención ha logrado un crecimiento medio, mientras que el grupo control, donde no se ha realizado intervención alguna se mantiene en los porcentajes (ver Tabla 4).

En el pretest: En esta dimensión se observa que los dos grupos han tenido experiencia laguna 
referente a gamificación en el aula. Ambos grupos requieren fortalecer el trabajo en el desarrollo de este aspecto (ver Tabla 5).

En el post test: El grupo experimental ha logrado un mayor desarrollo en la técnica de gamificación en el aula, mientras que el grupo control se mantiene en los porcentajes iniciales. En el grupo control se observa que el 44,12\% permanece con deficiencias. El $41,18 \%$ ha mejorado en su desarrollo $y$ solo el $9,37 \%$ se mantiene en el nivel alto. A diferencia del grupo en el que se ha intervenido, donde $56,25 \%$ se encuentra en el nivel medio y el $28,12 \%$ ha alcanzado el nivel alto (ver Tabla 5).

\section{DISCUSIÓN}

Las tecnologías se insertan cada vez más en diferentes contextos de la sociedad y la vida cotidiana de las personas, por lo que las escuelas no desconocen esta nueva cultura, que es digital. En entornos educativos, se puede ver que esta cultura digital está influyendo en la formación de los estudiantes porque nacen en este momento de cambios tecnológicos acelerados y utilizan estas tecnologías en su vida diaria.

Sin embargo, los estudiantes generalmente no experimentan en las escuelas, particularmente las públicas, clases con estrategias y recursos de tecnologías contemporáneas de comunicación e información porque, aun así, la enseñanza tradicional es lo que prevalece en la enseñanza.

Los estudiantes ciertamente aspiran a clases diferentes de las tradicionales, más dinámicas y más cercanas a la realidad en la que viven, por lo que depende del maestro buscar su mejora profesional y actualizarse a través de cursos de capacitación que le permitan utilizar metodologías y estrategias de enseñanza. para calificar su práctica docente, a fin de satisfacer los deseos expresados por los estudiantes

El objetivo de esta investigación fue demostrar como la aplicación de la gamificación personalizada fortalecerá el aprendizaje significativo de la asignatura Matemática en los estudiantes de bachillerato de la ciudad de Guayaquil. Debido a que involucra elementos de juegos en contextos que están ideados para ser serios. La gamificación se caracteriza principalmente por el involucramiento en los factores afectivos de quienes participan en procesos de aprendizajes gamificados. Se puede considerar de mayor importancia el aprendizaje experiencial, la competencia, la autonomía y la tolerancia al error.

\section{CONCLUSIONES}

En el presente informe de investigación presentamos las siguientes conclusiones:

- La implementación de técnicas de gamificación en el aula virtual causó efectos en el aprendizaje significativo de las matemáticas en los estudiantes de bachillerato, lo cual se observa en los puntajes obtenidos por parte del grupo de control frente al grupo experimental.

- La utilización de plataformas virtuales gamificadas ha demostrado su eficacia no solo en el ámbito empresarial sino también en el ámbito educativo, las mismas que sirven como motivador intrínseco y extrínseco para los estudiantes.

- Los hallazgos atestiguan el hecho de que las lecciones de matemáticas combinadas y gamificadas pueden garantizar el crecimiento intelectual, físico, perceptivo, social y cognitivo de los estudiantes. Estos son importantes para el 
proceso de pensamiento, así como para promover

la conciencia ambiental y social.

- La estrategia de enseñanza puede ser un punto de partida para nuevos estudios e investigaciones en los procesos de enseñanza y aprendizaje de las Matemáticas, para mejorarla.

\section{RECOMENDACIONES}

Existen pocos estudios sobre qué elementos de la gamificación serían beneficiosos en estos casos $y$, con base en los datos obtenidos en este informe, creo que la investigación de elementos que promueven la motivación intrínseca como: narrativa, escenario y personajes puede tener un impacto positivo. Además, recomiendo expandir esta investigación con diferentes grupos de edad y contextos sociales, para identificar diferencias y similitudes en la producción de los estudiantes y comprender cómo podemos mejorar el proceso de gamificación en las aulas. Me gustaría enfatizar que el papel del investigador en el proceso de enseñanza y aprendizaje con la gamificación es esencial. En esta investigación, se instruyó al maestro en cada etapa, pero, si es posible, recomiendo que el investigador instruya a la clase y participe en el proceso de gamificación con los estudiantes, hablando, cuestionando y proponiendo nuevos enfoques. El hecho de que el investigador ya tenga contacto con la gamificación y conozca su mecánica, dinámica y estética, facilita la interacción y puede promover nuevas preguntas.

\section{REFERENCIAS}

Alves, F. (20I5). Gamificación: crear experiencias de aprendizaje atractivas. DVS Editora.
Andrade, F., Mizoguchi, R., \& Isotani, S. (20I6). The Bright and Dark Sides of Gamification. https://doi.org/I0.1007/978-3-319-39583-8_ I7

Ausubel, D. P. (1976). Psicología educativa. Un punto de vista cognoscitivo. México: Ed. Trillas.

Barata, G., Gama, S., \& Gonçalves, D. (20I3). Melhorando o Ensino universitário com a Gamificação.

Borges, S., Durelli, V., Reis, H., \& Isotani, S. (20I4). A Systematic Mapping on Gamification Applied to Education. https://doi.org/ I0.1 | 45/2554850.2554956

Borges, S., Durelli, V., Reis, H., Bittencourt, I., Mizoguchi, R., \& Isotani, S. (octubre de 2017). Selecting Effective Influence Principles for Tailoring Gamification-Based Strategies to Player Roles. https://doi.org//0.5753/cbie.sbie.2017.857

Burke, B. (20I5). Gamificar: cómo la gamificación motiva a las personas a hacer cosas extraordinarias. Sao Paulo: DVS editora.

Burke, B. (20I5). Gamify Cómo la gamificación motiva a las personas a hacer cosas extraordinarias. DVS Editora.

Carrasco, S. (2015). Metodología de la investigación científica: pautas metodológicas para diseñar y elaborar el proyecto de investigación. Lima, Perú: San Marcos.

Chou, Y. (20I6). Actionable Gamification: beyond points, badges and leaderboards. Revista Internacional de Organizaciones, I37-I44. doi: $10.17345 /$ riol 8

Clason, D., \& Dormody, T. (1994). Analyzing Data Measured by Individual Likert-Type Items. Journal of Agricultural Education 35 (4), 31-35. https://doi.org// 0.5032/jae/ | 994.0403 |

Deterding, S. (20I2). Gamification: designing for motivation. Interactions, New York, 19, I4-I7.

Dichev, C., \& Dicheva, D. (20I7). Gamifying education: what is known, what is believed and what remains uncertain: a critical review. https://doi.org//0.1186/s4I239-017-0042-5

Fedoce, R., \& Squirra, S. (20I I). Tecnología móvil y potencial de comunicación en educación. Rio de Janeiro: Logos.

Hamari, J., Koivisto, J., \& Sarsa, H. (20I4). Does Gamification Work? - A Literature Review of Empirical Studies on Gamification. 47th Hawaii International Conference on System Science, 30253034. https://doi.org/I0.1 I09/HICSS.2014.377

Kapp, K. M. (2012). The gamification of learning and instruction: game-based methods and strategies for training and education. San Francisco, CA: Pfeiffer. 
Klock, A., de Carvalho, M., Eduardo Rosa, B., \& Gasparini, I. (20I5). Análisis de técnicas de gamificación en entornos virtuales de aprendizaje. ENOTE - Revista Novas Tecnologias na Educação. I2, I- 10. https://doi.org//0.22456/1679-1916.53496

Likert, R. (1932). Technique for the Measurement of Attitudes. New York.

Lozada-Ávila, C., \& Betancur-Gómez, S. (2017). La gamificación en la educación superior: una revisión sistemática. Revista Ingenierías Universidad de Medellín, 16 (3I) https://doi.org/10.22395/rium.v16n3la5

Mattar, J. (2017). Metodologias ativas para a educação presencial, blended e a distância. São Paulo: Artesanato Educacional.

Moreira, M. A. (1997). Aprendizagem Significativa: um conceito subyacente. Universidad de Burgos.

Moscardini, T., Monticelli, J., \& Velloso, M. (20I5). Nivel de utilización de m-learning (aprendizaje móvil) por estudiantes de pregrado: una encuesta. Nivel de utilización de m-learning (aprendizaje móvil) por estudiantes de pregrado: una encuesta, 53-7I. https://doi.org/l0.18316/23/6-5537.15.10

Nah, F. F.-H., Zeng, Q., Telaprolu, V. R., Ayyappa, A. P., \& Eschenbrenner, B. (20/4). Gamification of Education: A Review of Literature. $\mathrm{HClB} / \mathrm{HCll}$ 20I 4, LNCS 8527, 40I-409.

Ñaupas, H., Mejía, E., Novoa, E., \& Villagómez, A. (20I4). Metodología de la investigación científica y asesoramiento de tesis (2a ed.). Bogotá, Colombia: Xpress Estudio Gráfico y Digital S.A.

Pedro, Z., Lopes, A., Prates, B., Vassileva, J., \& Isotani, S. (20I5). Does gamification work for boys and girls?: An exploratory study with a virtual learning environment. Proceedings of the 30th Annual ACM Symposium on Applied Computing 214219. Salamanca, Spain: ACM.

Sainz, C. (20I5). Matemáticas a través del juego (gamificación). Logroño: Universidad de La Rioja.

Santaella, L. (20I3). Desafíos de la ubicuidad para la educación. Revista Ensino Superior Unicamp. Campinas, 9, 19-28.

Silva, A., \& Vilhegas, V. (20I3). IHC em dispositivos móveis - análise do aplicativo whatsapp. ETIC ENCONTRO DE INICIAÇÃO CIENTÍFICA. 9 (9).

Torres, A. I., \& Lázaro, D. (20I5). El proceso de gamificación en el aula: las matemáticas en educación infantil. Madrid: Grim.
Ventura, M. (20I3). Videojuegos, remix, conceptos transmedia para un paradigma emergente en plataformas digitales. Universidad de Aveiro, Programa de Doctorado Información y Comunicación sobre Plataformas Digitales. Universidad de Oporto.

Werbach, K., \& Hunter, D. (20I2). For the win: how game thinking can revolutionize your business. Philadelphia: Wharton Digital Press.

Zichermann, G., \& Cunningham, C. (20II). Gamification by Design. Canada: O’Reilly Media. 